\title{
Original
}

\section{Prevalence and clinical characteristics of primary aldosteronism in Japanese patients with type 2 diabetes mellitus and hypertension}

\author{
Kunitaka Murase, Ryoko Nagaishi, Hiromasa Takenoshita, Takashi Nomiyama, Yuko Akehi and \\ Toshihiko Yanase
}

Department of Endocrinology and Diabetes Mellitus, Faculty of Medicine, Fukuoka University, Fukuoka 814-0180, Japan

\begin{abstract}
The prevalence of primary aldosteronism (PA) is around 3-15\% in patients with hypertension. Hypertension is a frequent complication of type 2 diabetes mellitus (DM) because of the close etiological relationship between these two diseases. However, the possibility of PA in patients with DM and hypertension is often overlooked and the prevalence of PA in patients with DM and hypertension in Japan is unknown. We enrolled 124 hospitalized patients with both DM and hypertension. PA was diagnosed according to the modified criteria for Japanese patients. We examined the prevalence of PA and compared clinical characteristics between patients with and without PA. In another analysis of 43 patients with a confirmed diagnosis of PA, we compared the characteristics of patients with and without DM. Overall, 14/124 patients with DM and hypertension (11.3\%) were diagnosed with PA. Multivariate logistic regression showed that the duration of DM was significantly shorter in the PA group. Fisher's direct probability test revealed that history of hypertension before the diagnosis of DM was a significant factor in patients with PA. Treatment with an angiotensin II receptor blocker (ARB) did not affect the diagnosis of PA in these patients. Among 43 patients with PA, those with DM were significantly older and the delay to the diagnosis of PA was significantly longer compared with patients without DM. In conclusion, almost $10 \%$ of patients with DM and hypertension actually have PA. More extensive screening for PA is recommended in patients with DM and hypertension, regardless of the use of ARBs.
\end{abstract}

Key words: Primary aldosteronism, Type 2 diabetes mellitus, Hypertension

PRIMARY ALDOSTERONISM (PA) is characterized by autonomic oversecretion of aldosterone from the adrenal gland, resulting in increased renal sodium uptake and potassium excretion. Patients with PA often show secondary hypertension and sometimes hypokalemia [1]. PA is usually caused by an adrenal adenoma, although other causes exist, including bilateral adrenal hyperplasia (idiopathic hyperaldosteronism), for example. The prevalence of PA in hypertensive patients is estimated to be around $3.3-15.0 \%$ in Japan and in other countries [2-5]. Therefore, it seems that clinicians are increasingly becoming more aware of PA as a cause of hypertension. One reason for this increasing awareness may be widespread dissemination

Submitted Feb. 11, 2013; Accepted May 2, 2013 as EJ13-0060 Released online in J-STAGE as advance publication May 30, 2013

Correspondence to: Toshihiko Yanase, M.D., Department of Endocrinology and Diabetes Mellitus, School of Medicine, Fukuoka University, 7-45-1 Nanakuma, Jonan-ku, Fukuoka 8140180, Japan. E-mail: tyanase@fukuoka-u.ac.jp of the Japanese guidelines for PA published in 2011[1].

PA is also very commonly associated with impaired glucose tolerance, being present in almost $50 \%$ of individuals with IGT [6-8]. Although one of the underlying mechanisms, suppression of insulin secretion by low serum potassium is well recognized, aldosterone was also reported to directly inhibit insulin secretion from cultured pancreatic $\beta$ cells [9]. In addition, aldosterone induces IGT by stimulating the production of molecules that cause insulin resistance, including free fatty acids (FFA) and tumor necrosis factor, from adipocytes [10].

It was reported that $62 \%$ of patients with type 2 diabetes mellitus (DM) also have hypertension [11]. The coexistence of hypertension and DM accelerates the course of microvascular and macrovascular disease [12]. In DM, hyperinsulinemia caused by insulin resistance increases renal sodium uptake [13] and sympathetic nerve activity [14], which induce hypertension. The progression of diabetic nephropathy and atherosclerosis is also thought to be associated with the high 
prevalence of hypertension in patients with DM [12]. However, the prevalence of PA in patients with both DM and hypertension in Japan is unknown. In most patients with both DM and hypertension, the possibility of having PA is often overlooked, probably because $\mathrm{DM}$ and hypertension are well known to coexist. The frequent use of antihypertensive drugs, particularly angiotensin II receptor blockers (ARBs), in patients with $\mathrm{DM}$ and hypertension may also contribute to delays in performing screening tests for PA.

Three studies have reported the coexistence of PA and $\mathrm{DM}$ as a result of screening of hypertensive patients. In one study, the prevalence of PA was $14 \%$ in diabetic subjects $(n=100)$ with poorly controlled hypertension taking more than three antihypertensive drugs [15]. In that study, most subjects were African-Americans (93\%). In another study of 100 consecutive Asian patients with type $2 \mathrm{DM}$ with difficult to control or resistant hypertension, $13 \%$ of patients were diagnosed as PA [16]. More recently, in a study of 232 Chinese hypertensive patients, PA was found in 14-23\%, 2.3-23.3\%, and $9.1 \%$ of patients with DM, impaired glucose tolerance, and impaired fasting glucose, respectively [17]. In these studies, plasma aldosterone concentration (PAC)/ plasma renin activity (PRA) ratio (ARR) $\geq 200$ [17], $\geq 300$ [15], or $\geq 550$ [16] and lack of PAC suppression following administration of $2 \mathrm{~L}$ of $0.9 \%$ saline over 4 $\mathrm{h}$ were used to screen for and confirm the diagnosis of PA. However, no such studies have been conducted in Japan. Furthermore, the clinical characteristics, including the sequence of the diagnosis of hypertension and $\mathrm{DM}$, or the impact of ARBs on ARR in screening were not fully examined in the previous studies [15-17].

In this study, to determine the prevalence of PA in Japanese patients with DM and hypertension, we performed a retrospective analysis of 124 patients whose ARR was assessed. We found that $11.3 \%$ of these patients had PA, which was close to that observed among general hypertensive patients. In this report, we compared the clinical characteristics of patients with $\mathrm{DM}$ and hypertension with or without PA.

\section{Methods}

\section{Subjects and diagnosis of $P A$}

This research project was approved by the institutional board review on clinical research. Of 585 cases manifesting both DM and hypertension who were hospitalized in our department between April 1, 2009, and August 31, 2012, the PAC/PRA ratio (ARR) was assessed in 124 patients, who were included in this study because of their stable general condition. The remaining 461 patients had clinical disorders (e.g., diabetic ketosis, hyperosmolar non-ketotic syndrome, severe diabetic complications, severe infections, the need for rapid blood glucose control before operation, fragility, and a bedridden state) that meant they were unsuitable for inclusion in this study. Of the 124 patients, 79 were men $(63.7 \%)$ and their clinical characteristics are shown in Table 1. Hypertension was defined as systolic pressure $\geq 140 \mathrm{mmHg}$ and/or diastolic pressure $\geq 90$ $\mathrm{mmHg}$ according to the guidelines of the Japan Society of Hypertension 2009 [18]. Type 2 DM was defined according to the guidelines of the Japan Diabetes Society 2012 [19]. Insulin was used in 40/124 patients with DM. Oral hypoglycemic agents were also used, including sulfonylureas (SUs) in 27 patients, biguanides in 24 patients, $\alpha$-glucosidase inhibitors ( $\alpha$-GIs) in 10 patients, thiazolidinediones (pioglitazone) in 6 patients, glinides in 4 patients, and dipeptidyl peptidase- 4 inhibitors in 28 patients. Because it is unethical and unsafe to discontinue prescribed therapies, ARR was measured while the patients were using their antihypertensive and antidiabetic drugs. The antihypertensive drugs used at the measurement of ARR are shown in Table 2. If $A R R$ was $\geq 200$, the antihypertensive medications, including ARBs, angiotensin converting enzyme inhibitors (ACEI), $\beta$-blockers, and diuretics, were stopped and only calcium channel blockers (CCB), mostly nifedipine (10-20 mg/day) were administered for 3-5 days. The following tests were then performed in patients with an $A R R \geq 200$. PA was diagnosed in patients with $A R R \geq 200$ and who had positive outcomes for at least two of the following three tests: upright furosemideloading test, captopril challenge test, and rapid adrenocorticotropic hormone (ACTH) loading test. In the upright furosemide-loading test and the captopril-challenge test, positive outcomes were defined as PRA $<2.0$ $\mathrm{ng} / \mathrm{mL} \cdot \mathrm{h}$ or $\mathrm{ARR} \geq 200$, respectively, according to Japan Endocrine Society guidelines [1]. In addition to these guidelines, the usefulness of the ACTH loading test has been emphasized and is commonly performed in Japan $[20,21]$. In the rapid ACTH loading test, serum PAC $(\mathrm{pg} / \mathrm{mL}) /$ cortisol $(\mu \mathrm{g} / \mathrm{dL}) \geq 8.5$ were considered positive outcomes for PA [20].

Adrenal venous sampling (AVS) was performed by expert radiologists according to our previously reported method [22]. Briefly, synthetic ACTH (1-24 ACTH) 
Table 1 Clinical characteristics of 124 patients with DM and hypertension with or without PA

\begin{tabular}{|c|c|c|c|c|c|c|c|}
\hline & & Total & & PA & & Non PA & $t$-test, Fisher \\
\hline & $\mathrm{n}$ & Mean \pm S.D. & $\mathrm{n}$ & Mean \pm S.D. & $\mathrm{n}$ & Mean \pm S.D. & $P$ value \\
\hline Age & 124 & $63.01 \pm 12.08$ & 14 & $65.57 \pm 8.01$ & 110 & $62.68 \pm 12.49$ & 0.402 \\
\hline Sex (male/female) & 124 & $79 / 45$ & 14 & $8 / 6$ & 110 & $71 / 39$ & 0.572 \\
\hline Duration of DM (yr) & 120 & $12.84 \pm 11.3$ & 14 & $5.21 \pm 6.51$ & 106 & $13.73 \pm 11.43$ & 0.007 \\
\hline Duration of HT (yr) & 105 & $11.55 \pm 11.48$ & 14 & $13.15 \pm 11.60$ & 91 & $11.14 \pm 11.52$ & 0.582 \\
\hline BMI $\left(\mathrm{kg} / \mathrm{m}^{2}\right)$ & 122 & $25.68 \pm 4.96$ & 14 & $23.95 \pm 2.37$ & 108 & $25.90 \pm 5.16$ & 0.21 \\
\hline Systric BP (mmHg) & 124 & $142.4 \pm 22.45$ & 14 & $142.64 \pm 22.13$ & 110 & $142.35 \pm 22.59$ & 0.964 \\
\hline Diastric BP (mmHg) & 124 & $79.84 \pm 13.59$ & 14 & $79.64 \pm 14.94$ & 110 & $79.86 \pm 13.48$ & 0.549 \\
\hline Number of antihypertensive drug & 124 & $1.34 \pm 1.24$ & 14 & $1.75 \pm 1.49$ & 110 & $1.27 \pm 1.21$ & 0.214 \\
\hline $\mathrm{PAC}(\mathrm{pg} / \mathrm{mL})$ & 124 & $87.11 \pm 66.94$ & 14 & $167.9 \pm 84.06$ & 110 & $76.83 \pm 57.17$ & 0.0001 \\
\hline PRA (ng/mL·hr) & 124 & $2.16 \pm 3.80$ & 14 & $0.26 \pm 0.18$ & 110 & $2.40 \pm 3.97$ & 0.001 \\
\hline ARR & 124 & $200.50 \pm 432.47$ & 14 & $936.49 \pm 962.48$ & 110 & $100.47 \pm 100.88$ & 0.0062 \\
\hline $\mathrm{Na}(\mathrm{mEq} / \mathrm{L})$ & 124 & $139.68 \pm 3.52$ & 14 & $140.9 \pm 4.14$ & 110 & $139.52 \pm 3.43$ & 0.350 \\
\hline $\mathrm{K}(\mathrm{mEq} / \mathrm{L})$ & 124 & $4.13 \pm 0.48$ & 14 & $3.82 \pm 0.49$ & 110 & $4.17 \pm 0.47$ & 0.010 \\
\hline $\mathrm{Cr}(\mathrm{mg} / \mathrm{dL})$ & 124 & $1.22 \pm 1.09$ & 14 & $0.88 \pm 0.32$ & 110 & $1.27 \pm 1.14$ & 0.215 \\
\hline eGFR $\left(\mathrm{mL} / \mathrm{min} / 1.73 \mathrm{~m}^{2}\right)$ & 124 & $62.01 \pm 26.70$ & 14 & $65.26 \pm 17.47$ & 110 & $61.60 \pm 27.69$ & 0.631 \\
\hline Microalbuminuria (mg/g/Cr) & 54 & $63.08 \pm 108.25$ & 9 & $42.60 \pm 92.39$ & 45 & $67.17 \pm 111.63$ & 0.539 \\
\hline FBS (mg/dL) & 124 & $159.20 \pm 58.48$ & 14 & $115.29 \pm 21.21$ & 110 & $164.79 \pm 59.38$ & 0.0001 \\
\hline HbA1c (NGSP) (\%) & 124 & $8.61 \pm 1.92$ & 14 & $7.26 \pm 1.43$ & 110 & $8.79 \pm 1.91$ & 0.005 \\
\hline Urine $C$ peptide ( $\mu \mathrm{g} /$ day) & 114 & $74.99 \pm 57.36$ & 10 & $76.49 \pm 32.43$ & 104 & $74.84 \pm 59.31$ & 0.932 \\
\hline
\end{tabular}

$P$ values were determined using Student's independent-samples $t$-test and were considered to be statistically significant at $P<0.05$. $\mathrm{DM}$, diabetes mellitus; PA, primary aldosteronism; PAC, plasma aldosterone concentration; PRA, plasma renin activity; ARR, aldosterone/renin ratio

Table 2 Types of antihypertensive drugs at the measurement of ARR in patients with PA with or without DM

\begin{tabular}{lccc}
\hline & Total (\%) & PA (\%) & Non PA (\%) \\
\hline ARB & $58(46.8)$ & $5(35.7)$ & $53(48.1)$ \\
CCB & $58(46.8)$ & $11(78.6)$ & $47(42.7)$ \\
Diuretic drug & $11(8.9)$ & $1(7.1)$ & $10(9.1)$ \\
ACE-I & $2(1.6)$ & $1(7.1)$ & $1(0.9)$ \\
$\alpha$-blocker & $10(7.8)$ & $4(28.6)$ & $6(5.5)$ \\
$\beta$-blocker & $7(5.6)$ & $3(21.4)$ & $4(3.6)$ \\
Selective aldosteron blocker & $1(0.8)$ & $1(0.9)$ & $0(0)$ \\
Direct renin blocker & $2(1.6)$ & $0(0)$ & $2(1.8)$ \\
\hline
\end{tabular}

$\overline{\mathrm{ARR}}$, aldosterone/renin ratio; DM, diabetes mellitus; PA, primary aldosteronism; ARB, angiotensin II receptor blocker; CCB, calcium channel blocker; ACE-I, angiotensin-converting enzyme inhibitor

was intravenously administered at $50 \mu \mathrm{g} / \mathrm{h}$ from $60 \mathrm{~min}$ before AVS until the end of AVS. Blood samples were taken from the proximal inferior vena cava (IVC), left adrenal vein, right adrenal vein, and distal IVC. Adrenal vein cannulation was considered successful if the adrenal vein cortisol level was $\geq 200 \mu \mathrm{g} / \mathrm{dL}$ or if the adrenal vein/IVC cortisol ratio was $\geq 5$. The patient was diagnosed with aldosterone hypersecretion (i.e., PA) if the PAC was $\geq 14,000 \mathrm{pg} / \mathrm{mL}$ in either or both of the adrenal veins. Lateralization was considered if the aldosterone to cortisol ratio $(\mathrm{A} / \mathrm{C})$ from one adrenal gland was $\geq 3$ times the ratio of the other adrenal gland (lateralization ratio [LR]) and the $\mathrm{A} / \mathrm{C}$ in the contralateral adrenal vein was lower than the $\mathrm{A} / \mathrm{C}$ in the IVC (contralateral ratio
[CR]), as previously described [23].

Although AVS was only performed in 22 patients between April 1, 2009 and August 31, 2012, we calculated sensitivity, specificity, and accuracy of our diagnostic criteria of PA by discriminate analysis based on positive outcomes for at least two of the following three tests: upright furosemide-loading test, captopril challenge test, and rapid ACTH loading test. The sensitivity, specificity, and accuracy were $95.0 \%, 100 \%$ and $95.2 \%$, respectively, for satisfying the criteria of both the upright furosemide-loading test and the captoprilchallenge test; were $95.2 \%, 100 \%$ and $95.5 \%$, respectively, for satisfying the criteria of both the upright furosemide-loading test and the rapid ACTH test; and 
were $90.5 \%, 100 \%$, and $90.9 \%$, respectively, for satisfying the criteria of both the captopril-challenge test and the rapid ACTH test. The sensitivity, specificity, and accuracy for satisfying the criteria of all three tests were $90.5 \%, 100 \%$, and $90.9 \%$, respectively.

Next, we compared the clinical characteristics of patients with and without PA, focusing on their age, sex, body mass index (BMI), duration of hypertension, duration of DM, type of antihypertensive drugs, PRA, PAC, serum creatinine, serum $\mathrm{Na}$, serum $\mathrm{K}$, fasting blood sugar (FBS), HbA1c (National Glycohemoglobin Standardization Program [NGSP] units), and 24-h urine C-peptide immunoreactivity (CPR). FBS was measured using an automatic glucose monitor (GA-1170; ARKRAY, Inc., Kyoto, Japan) based on the amperometric method. The effects of using different first-line antihypertensive drugs in patients with DM, including ARBs, ACEIs, and CCBs [18, 24, 25] on ARR and the diagnosis of PA were also investigated.

In 43 patients with a confirmed diagnosis of PA who were hospitalized at our department between April 1, 2009, and August 31, 2012, we compared the clinical characteristics of those with and without DM. The sequence of the diagnosis of DM and hypertension was obtained from the clinical records based on the patient's medical history.

\section{Statistical analysis}

All data are expressed as means \pm standard deviation. Univariate analyses were performed using Student's independent-samples $t$-test. For multivariate analyses, we used logistic regression or Fisher's test, as appropriate. Values of $P<0.05$ were considered statistically significant.

\section{Results}

\section{Prevalence of $P A$ in patients with $D M$ and hypertension}

In the observation period, 585 patients with both DM and hypertension were hospitalized at our department. ARR was measured in 124 of these patients, and their characteristics are summarized in Table 1. The mean age was $63.01 \pm 12.08$ years old. The mean durations of DM and hypertension were $12.84 \pm 11.3$ years and $11.55 \pm 11.48$ years, respectively. The mean systolic and diastolic blood pressures (BP) were 142.4 \pm 22.45 $\mathrm{mmHg}$ and $79.84 \pm 13.59 \mathrm{mmHg}$, respectively. Most of the 124 patients had poor glycemic control because the mean HbA1c (NGSP) level was $8.61 \pm 1.92 \%$.
ARR was $\geq 200$ in $23 / 124$ patients. Of these, six had low PRA and low PAC $(<70 \mathrm{pg} / \mathrm{mL})$ as a result of severe diabetic neuropathy, giving the appearance of $A R R \geq 200$, and were excluded from further analyses. The other 17 patients underwent loading or suppression tests to confirm the diagnosis of PA and 14 patients were positive on more than two tests. Of these 14 patients, nine patients were positive on all three tests and three patients were positive on two tests. The other two patients had ARR values of 1390 and 917, respectively, and both satisfied the criteria of the upright furosemide-loading test and the captopril challenge test. However, neither of these patients wished to undergo the ACTH loading test. Therefore, these 14 patients were ultimately diagnosed with PA, corresponding to $11.3 \%$ of the subjects with DM and hypertension.

\section{Clinical characteristics of patients with DM and hypertension with $P A$ or without $P A$}

Next, we compared the characteristics of patients with DM and hypertension together with PA $(\mathrm{N}=14)$ or without PA $(\mathrm{N}=110)$ (Table 1). There were no significant differences in terms of age, sex, BP, BMI, or the number of antihypertensive drugs. However, duration of DM was significantly shorter in patients with $\mathrm{PA}(5.21 \pm 6.51$ years $)$ than in those without PA $(13.73 \pm 11.43$ years $)$ (Table 1). Multivariate logistic regression analysis showed that a shorter duration of DM, but no other variable, was significantly associated with the occurrence of PA (Table 3). On the other hand, the duration of hypertension was not significantly different between the two groups. The history of DM and hypertension could be assessed in 14 patients with PA and in 91 patients without PA. Hypertension was diagnosed before DM in 8/14 patients with PA, and in 17/91 patients without PA. DM was diagnosed before hypertension in 3/14 patients with PA, and in 43/91 patients without PA. Hypertension and DM were diagnosed at the same time in $3 / 14$ patients with PA and in 31/91 patients without PA. Fisher's direct probability test revealed that the difference in the sequence of diagnosis of HT and DM significantly contributed to the diagnosis of PA as patients with PA were significantly more likely than patients without PA to be diagnosed with hypertension before $\operatorname{DM}(P=0.013$; Fig. 1). The mean serum $K$ level was significantly lower in patients with PA than in those without PA (3.82 vs. 4.17 $\mathrm{mEq} / \mathrm{L}$ ). However, the serum $\mathrm{K}$ level was within the normal range $(>3.5 \mathrm{mEq} / \mathrm{L})$ in $13 / 14$ patients with $\mathrm{PA}$ (92.8\%); the other patient had a very low serum $\mathrm{K}$ level 
Table 3 Multivariate logistic regression analysis of factors associated with PA in patients with DM and hypertension ( $\mathrm{n}=124)$

\begin{tabular}{lcclcc}
\hline & Regression coefficient & S.E & $P$ value & Contribution & Odds ratio (95\% C.I) \\
\hline Sex & 0.415 & 0.776 & 0.5926 & 0 & $1.52(0.33-6.93)$ \\
Age & 0.008 & 0.038 & 0.8327 & 0 & $1.01(0.93-1.09)$ \\
BMI & -0.194 & 0.109 & 0.768 & -0.13 & $0.82(0.66-1.02)$ \\
Duration of DM & -0.095 & 0.047 & 0.0463 & -0.18 & $0.91(0.82-0.99)$ \\
Duration of HT & 0.045 & 0.033 & 0.1791 & 0 & $1.05(0.97-1.12)$ \\
Systolic BP & 0.007 & 0.022 & 0.7528 & 0 & $1.01(0.96-1.05)$ \\
Diastolic BP & -0.02 & 0.041 & 0.5421 & 0 & $0.98(0.89-1.06)$ \\
\hline
\end{tabular}

DM, diabetes mellitus; PA, primary aldosteronism; HT, hypertension; BP, blood pressure In the case of sex, male and female was defined as 1 and 2 , respectively.

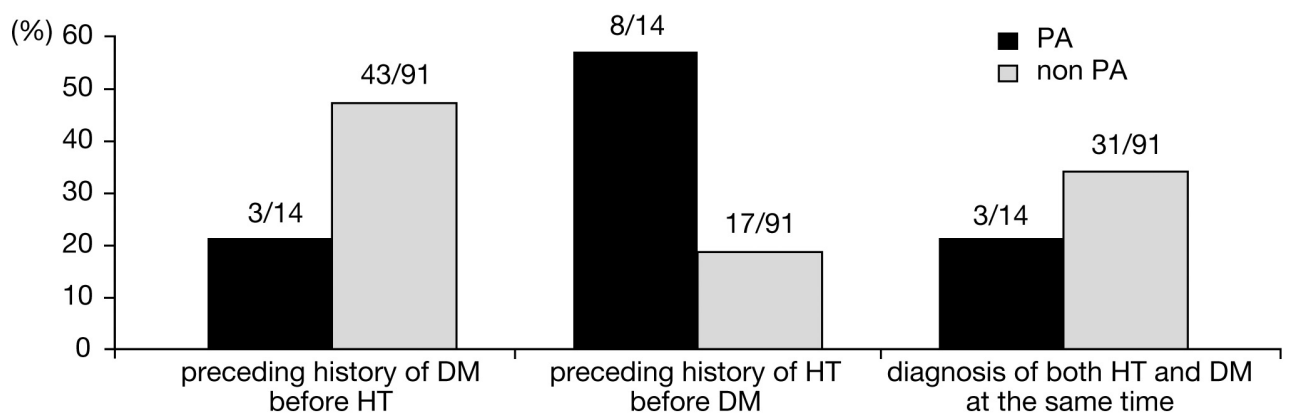

Fig. 1 Comparison of the sequence of diagnosis of hypertension (HT) and diabetes mellitus (DM) in patients with or without primary aldosteronism. black column, patients with PA; dark column, patients without PA. Fisher's direct probability test revealed that the sequence of diagnosis of HT and DM significantly contributed to the diagnosis of PA $(P=0.013)$.

$(2.3 \mathrm{mEq} / \mathrm{L})$. Regarding other factors, FBS and HbA1c were significantly lower in patients with PA than in those without PA, but 24-h urinary CPR was not significantly different between the two groups (Table 1).

\section{Effects of antihypertensive drugs on ARR}

The effects of antihypertensive drugs on PRA, PAC, ARR, and the diagnosis of PA were investigated. The types of antihypertensive drugs used at the measurement of ARR in patients with and without PA are listed in Table 2. Because most of the patients were prescribed with an ARB and/or CCB, we focused on these two classes of drugs. The values of PAR, PAC, and ARR differed, although not significantly, between those treated with or without an ARB in all 124 patients, and between those with or without PA (Table 4). Fisher's analysis confirmed that treatment with an ARB or CCB did not affect ARR. The $P$ values in these analyses for the use of an ARB or CCB were 0.941 and 0.17 , respectively.

\section{Further examination and the treatment of hyperten- sion in $P A$ patients}

Of 14 diabetic patients with PA, 12 underwent AVS. Based on the results of this test, four patients were diagnosed with bilateral aldosterone-producing disorders and eight patients were diagnosed with unilateral aldosterone-producing disorders. Among eight patients with unilateral adrenal disorders, abdominal computed tomography found no evidence of an adrenal tumor in two patients, contralateral adrenal tumors in two patients, and an adrenal tumor located in the affected adrenal gland in four patients. Only one patient had undergone unilateral adrenalectomy, and an adrenal adenoma was pathologically confirmed in this patient. Although her hypertension improved after adrenalectomy, she needed to continue her antihypertensive drugs. Hypertension was well controlled in the other 13 patients $(<140 / 90 \mathrm{mmHg})$, mostly with CCBs, eplerenone, or both.

\section{Clinical characteristics of patients with PA with DM or without DM}

Among the 43 patients with a confirmed diagnosis of PA, 15 had DM (34.9\%) and 28 did not. The clinical characteristics of both groups are summarized in Table 5. The patients with DM were significantly older $(66.45 \pm 8.43$ vs. $55.07 \pm 10.53$ years $)$ and their diagnosis of DM was considerably delayed $(17.44 \pm 11.28$ 
Table 4 Effect of ARBs on PRA, PAC, and ARR in patients with DM and hypertension

\begin{tabular}{lcccc}
\hline & & ARB $(+)$ & ARB $(-)$ & $P$ value \\
\hline \multirow{4}{*}{ Total } & $\mathrm{n}$ & 58 & 66 & \\
& $\mathrm{PRA}( \pm \mathrm{SD})$ & $2.77( \pm 4.57)$ & $1.62( \pm 2.89)$ & 0.095 \\
& $\mathrm{PAC}( \pm \mathrm{SD})$ & $76.90( \pm 65.2)$ & $96.08( \pm 67.7)$ & 0.118 \\
& ARR $( \pm \mathrm{SD})$ & $128.9( \pm 190.7)$ & $246.4( \pm 545.9)$ & 0.122 \\
\hline \multirow{4}{*}{$\mathrm{PA}$} & $\mathrm{n}$ & 5 & 9 & \\
& $\mathrm{PRA}( \pm \mathrm{SD})$ & $0.24( \pm 0.05)$ & $0.28( \pm 0.22)$ & 0.720 \\
& PAC $( \pm \mathrm{SD})$ & $163.4( \pm 73.5)$ & $170.4( \pm 93.6)$ & 0.887 \\
& ARR $( \pm \mathrm{SD})$ & $666.1( \pm 207.3)$ & $1087( \pm 1188.6)$ & 0.456 \\
\multirow{4}{*}{ Non PA } & $\mathrm{n}$ & 53 & 57 & \\
& PRA $( \pm \mathrm{SD})$ & $3.00( \pm 4.71)$ & $1.83( \pm 3.06)$ & 0.123 \\
& PAC $( \pm \mathrm{SD})$ & $68.75( \pm 58.7)$ & $84.34( \pm 55.1)$ & 0.153 \\
& ARR $( \pm \mathrm{SD})$ & $78.22( \pm 78.6)$ & $113.7( \pm 112.6)$ & 0.060 \\
\hline
\end{tabular}

The effects were examined in all patients, and in patients with or without PA.

$P$ values were determined using Student's independent-samples $t$-test and were considered to be statistically significant at $P<0.05$.

$\mathrm{ARB}$, angiotensin II receptor blocker; DM, diabetes mellitus; PA, primary aldosteronism; PAC, plasma aldosterone concentration; PAR, plasma renin activity; ARR, PAC/PRA ratio

Table 5 Clinical profiles of patients with PA with or without DM

\begin{tabular}{|c|c|c|c|c|c|}
\hline & $\mathrm{n}$ & $\mathrm{DM}($ Mean \pm S.D $)$ & $\mathrm{n}$ & Non DM (Mean \pm S.D) & $P$ value \\
\hline Sex (male / female) & 15 & $8 / 7$ & 28 & $9 / 19$ & 0.766 \\
\hline Age (yr) & 15 & $66.45 \pm 8.43$ & 28 & $55.07 \pm 10.53$ & 0.0017 \\
\hline Duration of HT (yr) & 10 & $17.44 \pm 11.28$ & 25 & $7.55 \pm 9.87$ & 0.018 \\
\hline BMI $\left(\mathrm{kg} / \mathrm{m}^{2}\right)$ & 15 & $24.7 \pm 2.49$ & 27 & $22.48 \pm 2.56$ & 0.203 \\
\hline Number of antihypertensive drug & 15 & $1.80 \pm 1.54$ & 28 & $1.17 \pm 1.25$ & 0.067 \\
\hline Systric BP (mmHg) & 15 & $141.3 \pm 22.17$ & 28 & $130.4 \pm 15.25$ & 0.181 \\
\hline Diastric BP (mmHg) & 15 & $80.00 \pm 15.32$ & 28 & $80.56 \pm 10.66$ & 0.787 \\
\hline $\mathrm{PAC}(\mathrm{pg} / \mathrm{mL})$ & 15 & $180.6 \pm 104.6$ & 28 & $219.3 \pm 167.1$ & 0.310 \\
\hline PRA (ng/mL·hr) & 15 & $0.24 \pm 0.08$ & 28 & $0.27 \pm 0.14$ & 0.072 \\
\hline ARR & 15 & $822.5 \pm 491.8$ & 28 & $1080.5 \pm 1523.2$ & 0.981 \\
\hline $\mathrm{Cr}(\mathrm{mg} / \mathrm{dL})$ & 15 & $0.89 \pm 0.31$ & 28 & $0.72 \pm 0.18$ & 0.042 \\
\hline $\mathrm{eGFR}\left(\mathrm{mL} / \mathrm{min} / 1.73 \mathrm{~m}^{2}\right)$ & 15 & $66.6 \pm 16.9$ & 28 & $77.10 \pm 19.48$ & 0.106 \\
\hline $\mathrm{Na}(\mathrm{mEq} / \mathrm{L})$ & 15 & $141.4 \pm 3.85$ & 28 & $141.7 \pm 1.44$ & 0.355 \\
\hline $\mathrm{K}(\mathrm{mEq} / \mathrm{L})$ & 15 & $3.82 \pm 0.52$ & 28 & $3.64 \pm 0.45$ & 0.097 \\
\hline HbA1c (NGSP) (\%) & 15 & $7.41 \pm 1.62$ & 15 & $5.59 \pm 0.17$ & 0.0004 \\
\hline The antihypertensive drug & $\mathrm{n}$ & DM (\%) & $\mathrm{n}$ & Non DM (\%) & \\
\hline $\mathrm{ARB}$ & 5 & $(33.3)$ & 8 & $(28.6)$ & $0.99^{\mathrm{a}}$ \\
\hline $\mathrm{CCB}$ & 12 & $(80)$ & 14 & $(50)$ & $0.45^{\mathrm{a}}$ \\
\hline Diuretic drug & 1 & (6.7) & 2 & (7.1) & \\
\hline ACE-I & 1 & (6.7) & 2 & $(7.1)$ & \\
\hline$\alpha$-blocker & 3 & (20) & 0 & $(0)$ & \\
\hline$\beta$-blocker & 2 & $(13.3)$ & 3 & $(10.7)$ & \\
\hline Selective aldosteron blocker & 1 & $(6.7)$ & 1 & (3.6) & \\
\hline
\end{tabular}

$P$ values were determined using Student's independent-samples $t$-test and were considered to be statistically significant at $P<0.05$. The types of antihypertensive drugs at the time of measurement of ARR in patients with PA with or without DM are also shown. PAC, plasma aldosterone concentration; PRA, plasma renin activity; ARR, PAC/PRA ratio; ARB, angiotensin II receptor blocker; CCB, calcium channel blocker; ACE-I, angiotensin-converting enzyme inhibitor a statistical value based on Fisher's probability test for the ARR determination between DM and non DM patients. 
vs. $7.55 \pm 9.87$ years) compared with patients without DM. HbA1c was also significantly different between the two groups. By contrast, other factors, including PRA, PAC, and ARR, were not significantly different between patients with and without DM. The effects of hypertensive drugs on ARR were also examined in this population. The types of antihypertensive drugs used at the first measurement of ARR in patients with and without PA are also listed in Table 5. As most of the patients were treated with an ARB and/or CCB, we focused on these classes of antihypertensive drugs. Fisher's analysis revealed no significant effects of ARBs or CCBs on ARR in patients with or without DM. The $P$ values in these analyses for the use of ARBs and CCBs were 0.99 and 0.45 , respectively (Table 5 ).

\section{Discussion}

PA is characterized by autonomic overproduction of aldosterone. It causes hypertension as well as oxidative stress, including oxidation of low-density lipoproteins and endothelial dysfunction, which promote atherosclerosis [26, 27]. Aldosterone also induces damage in several tissues, including the brain, heart, and kidney [24]. Drugs that inhibit aldosterone activity, such as mineralocorticoid receptor (MR) antagonists (e.g., spironolactone and eplerenone) show beneficial anti-oxidative and anti-atherosclerotic effects $[28,29]$. The results of the Randomized Aldactone Evaluation Study (RALES) were particularly remarkable in that the addition of a low dose of spironolactone $(25 \mathrm{mg})$ to an ACE, diuretic, and digitalis was associated with a dramatic $(30 \%)$ reduction in the overall mortality rate among patients with severe cardiac failure [30]. Accordingly, early diagnosis and treatment of PA is particularly important.

Hypertension in patients with PA was reported to be improved or cured by operative resection of the responsible unilateral adrenal adenoma in most patients [31], supporting the concept that surgical excision of the adenoma should be the first-line therapy, if possible. In patients with non-operable tumors or for those who cannot hope for surgery, antihypertensive drugs are usually used, and remarkable effects from drugs that inhibit aldosterone activity have been reported [32]. Additionally, some dihydropyridine CCBs, such as nifedipine, were reported to possess MR antagonist activity and effectively control BP in patients with PA [33]. Although the coexistence of DM and hyperten- sion is very common, only three studies have examined the prevalence of PA in such patients in other countries [15-17]. The overall prevalence of PA in patients with both DM and hypertension ranged from $13 \%$ to $24 \%$ in those studies [15-17]. However, only two of those studies focused on DM patients with resistant hypertension $[15,16]$. In the present study, we targeted subjects with DM and unselected hypertension, and found that $11.3 \%$ of patients with DM and hypertension actually had PA. Thus, the prevalence of PA in Japanese diabetic patients with hypertension is similar to that observed in other countries.

While many studies have determined the prevalence of PA in unselected hypertensive patients within institutions, the prevalence of PA in hypertensive patients is approximately $3.3-15.0 \%$ [2-5]. Therefore, the frequent coexistence of DM and hypertension might prompt clinicians to overlook PA as the most common secondary hypertension. If hypertension in patients with DM is secondary to DM, the prevalence of PA in patients with DM and hypertension may be lower than that in the general hypertensive population. Surprisingly, however, the prevalence of PA in our study and in other studies was almost the same as that in the hypertensive population. According to the National Health and Nutrition Examination Survey in 2003, only 53\% of individuals achieved the BP target of 140/90 $\mathrm{mmHg}$ [34]. The same study also showed that only $25 \%$ of patients with both DM and hypertension achieved the BP target of $<130 / 85 \mathrm{mmHg}$, indicating the difficulty of controlling hypertension in patients with DM [34]. Resistant hypertension is a condition in which BP cannot be adequately controlled, despite using more than three antihypertensive drugs. In a study of 1616 patients with resistant hypertension, 182 patients $(11.6 \%)$ were ultimately diagnosed with PA [35]. Based on these findings, it is essential that PA is promptly diagnosed to facilitate appropriate antihypertensive treatment, particularly in patients with resistant hypertension.

It has been suggested that PRA, PAC, and ARR can be affected by several kinds of antihypertensive drugs, sometimes leading to false-positive or falsenegative results of screening or diagnostic tests for PA. Antihypertensive drugs that may influence the reninangiotensin-aldosterone system (RAAS), including ARBs, ACEIs, aldosterone blockers, diuretics, and $\beta$-blockers, are not recommended during the screening of PA [1]. Instead, CCBs and $\alpha$-blockers, which have weaker effects on the RAAS, are recommended 
[1]. ARBs and ACEs are commonly used as first-line antihypertensive drugs in patients with DM because of their renoprotective effects and because they alleviate insulin resistance to some extent [18, 24, 25]. In this study, we examined the effects of various antihypertensive drugs, including ARBs on the diagnosis of PA. However, ARR was not significantly affected by the classes of antihypertensive drugs, including ARBs, in patients with or without PA defined as ARR $\geq 200$. Similar results were reported in several studies of general hypertensive populations [36, 37]. Even in one patient with DM and PA who was treated with eplerenone, the ARR was 3840 and all three tests were positive. This case was considered to be typical of PA. Nevertheless, we should not reject the possibility that some of the patients without PA in our study were incorrectly classified based on false-negative results of the tests for PA. Therefore, to reach a definitive conclusion in suspected or borderline patients, reexamination may be necessary after halting the administration of ARBs or ACEIs.

Antidiabetic drugs appear to have very little effect on ARR. To our knowledge, there have been very few reports showing clear effects of SUs, $\alpha$-GIs, biguanides, and glinides on the RAAS in humans. Intriguingly, pioglitazone was reported to suppress the angiotensin II-induced increase in aldosterone production from cultured $\mathrm{H} 295$ adrenocortical cancer cells, which occurred in combination with the suppression of CYP11B2 [38]. However, in patients with DM complicated with coronary disease, $15 \mathrm{mg}$ pioglitazone did not appear to affect the RAAS, as it did not alter PRA or PAC [39]. Although glucagon-like peptide-1 was recently reported to reduce proximal tubular sodium reabsorption, it did not affect PRA or PAC following its administration to young healthy males [40].

The RAAS cascade begins with renin secretion from renal juxtaglomerular cells in response to renal hypoperfusion, decreased sodium delivery, and sympathetic activation. Thus, patients with severe diabetic neuropathy are sometimes thought to have RAAS suppression, characterized by low PRA and PAC levels, because of reduced activity of the sympathetic nervous system $[25,41]$. In such circumstances, we must be careful when evaluating the ARR and the results of the loading or suppression tests. In our study, the mean PAC level in patients with PA was $167.9 \mathrm{pg} / \mathrm{mL}$. Because patients with $\mathrm{PAC} \geq 150 \mathrm{pg} / \mathrm{mL}$ were strongly suspected of having PA [42], the PAC in our study seems to indicate autonomic aldosterone secretion. The mean FBS and HbA1c levels in patients with PA were $115.3 \mathrm{mg} / \mathrm{dL}$ and $7.26 \%$ (NGSP), respectively, suggesting that the diabetic status of these patients was relatively mild. The relatively small effect of DM on the RAAS was unlikely to have a marked impact on the diagnosis of PA in our study. However, we cannot reject the possibility that some false-negative test results led to the incorrect classification of some patients as not having PA. Consequently, we think that the prevalence of PA (11.3\%) in patients with DM and hypertension in this study might slightly underestimate the true prevalence of PA.

Two of the 14 patients with DM did not undergo AVS. Although obesity-associated hyperaldosteronism [43] could result in a false-positive diagnosis of PA, it was unlikely in these two patients because their BMI was 19.6 and $23.4 \mathrm{~kg} / \mathrm{m}^{2}$.

A notable feature of the patients with PA, DM, and hypertension in our study is that they had a relatively shorter history of DM and most were diagnosed with hypertension before DM compared with the patients without PA. Although this was not apparent in all of the patients with PA, these differences were statistically significant based on the results of Fisher's test. If hypertension is secondary to DM, DM should develop either before or at the same time as hypertension. The greater tendency for hypertension to occur before DM in patients with PA rejects this possibility and highlights the relatively independent pathology of hypertension. This sequence of events in patients with PA also suggests that DM may be secondary to PA because PA is well known to induce IGT through various mechanisms [6-10]. Nevertheless, the unique medical history of patients with PA should prompt clinicians to consider the possibility of PA in patients with both $\mathrm{DM}$ and hypertension. Among patients with DM and hypertension, the serum potassium, FBS, and $\mathrm{HbAlc}$ levels were relatively lower in patients with PA than in those without PA. The lower mean FBS $(115.3 \mathrm{mg} / \mathrm{dL})$ and $\mathrm{HbA} 1 \mathrm{c}(7.26 \%)$ levels may also reflect the shorter duration of DM in patients with PA.

In an independent analysis of 43 patients with confirmed PA, we compared the characteristics between those with or without DM. Interestingly, the patients with DM were significantly older and the diagnosis of PA was considerably delayed compared with patients without DM. The delayed diagnosis of PA in patients with DM may be explained by the assumption that 
hypertension is probably secondary to DM, when, in fact, PA seems to be much more prevalent than expected among patients with DM and hypertension.

There are several limitations to our study including a relatively small number of the subjects and the fact that we only included hospitalized patients. Additionally, we cannot ignore the possibility of selection bias because some patients with hypertension and DM were not enrolled in this study owing to their general conditions and situations.

In conclusion, we found that $11.3 \%$ of patients with DM and hypertension have PA. Among patients with $\mathrm{DM}$, the development of hypertension before DM should prompt clinicians to suspect the possibility of PA as a cause of hypertension. Our results also suggest that there is no need to stop antihypertensive med- ications to reach the diagnosis of PA, except in sus$\mathrm{pected} /$ borderline cases requiring reexamination. More extensive screening for PA is highly recommended for patients with both DM and hypertension.

\section{Acknowledgements}

This research was partially supported by Intractable Disease Research Grant of Ministry of Health, Labour and Welfare, Japan (ID: 11103517).

\section{Disclosure}

The authors declare no conflicts of interest relevant to this work.

\section{References}

1. Nishikawa T, Omura M, Satoh F, Shibata H, Takahashi K, et al. (2011) Task Force Committee on Primary Aldosteronism, The Japan Endocrine Society. Guidelines for the diagnosis and treatment of primary aldosteronism--the Japan Endocrine Society 2009. Endocr J 58: 711-721.

2. Nishikawa T, Omura M, Saito J, Matsuzawa Y (2012) Primary aldosteronism: comparison between guidelines of the Japanese and the US Endocrine Society. Expert Rev Endocrinol Metab 7: 637-645.

3. Omura M, Saito J, Yamaguchi K, Kakuta Y, Nishikawa $\mathrm{T}$ (2004) Prospective study on the prevalence of secondary hypertension among hypertensive patients visiting a general outpatient clinic in Japan. Hypertens Res 27:193-202.

4. Rossi GP, Bernini G, Caliumi C, Desideri G, Fabris B, Porteri E, et al. (2006) prospective study of the prevalence of primary aldosteronism in 1,125 hypertensive patients. J Am Coll Cardiol 48: 2293-2300.

5. Gordon RD, Ziesak MD, Tunny TJ, Stowasser M, Klemm SA (1993) Evidence that primary aldosteronism may not be uncommon: $12 \%$ incidence among antihypertensive drug trial volunteers. Clin Exp Pharmacol Physiol 20: 296-298.

6. Reincke M, Meisinger C, Holle R, Quinkler M, Hahner $\mathrm{S}$, et al. (2010) Is primary aldosteronism associated with diabetes mellitus? Results of the German Conn's Registry. Horm Metab Res 42: 435-439.

7. Strauch B, Widimský J, Sindelka G, Skrha J (2003) Does the treatment of primary hyperaldosteronism influence glucose tolerance? Physiol Res 52: 503-506.

8. Corry DB, Tuck ML (2003) The effect of aldosterone on glucose metabolism. Curr Hypertens Rep 5: 106-109.

9. Pierluissi J, Navas FO, Ashcroft SJ (1986) Effect of adrenal steroids on insulin release from cultured rat islets of Langerhans. Diabetologia 29: 119-121.

10. Wada T, Ohshima S, Fujisawa E, Koya D, Tsuneki H, et al. (2009) Aldosterone inhibits insulin-induced glucose uptake by degradation of insulin receptor substate (IRS) 1 and IRS2 via a reactive oygen species-mediated pathway in 3T3-L1 adipocytes. Endocrinology 150: 1662-1669.

11. Iimura O (1996) Insulin resistance and hypertension in Japanese. Hypertension Res 19 suppl 1: S1-8.

12. Sowers JR, Epstein M, Frohlich ED (2001) Diabetes, hypertension, and cardiovascular disease: an update. Hypertension 37: 1053-1059.

13. DeFronzo RA (1981) The effect of insulin on renal sodium metabolism. A review with clinical implications. Diabetologia 21: 165-171.

14. Anderson EA, Hoffman RP, Balon TW, Sinkey CA, Mark AL (1991) Hyperinsulinemia produces both sympathetic neural activation and vasodilation in normal humans. J Clin Invest 87: 2246-2252.

15. Umpierrez GE, Cantey P, Smiley D (2007) Primary aldosteronism in diabetic subjects with resistant hypertension. Diabetes Care 30: 1699-1703.

16. Mukherjee JJ, Khoo CM, Thai AC (2010) Type 2 diabetic patients with resistant hypertension should be screened for primary aldosteronism. Diab Vasc Dis Res 7: 6-11.

17. Li N, Wang M, Wang H, Zhang D, Wang X, et al. (2013) Prevalence of primary aldosteronism in hypertensive subjects with hyperglycemia. Clin Exp Hypertens 35: 
175-182.

18. Ogihara T, Kikuchi K, Matsuoka H, Fujita T, Higaki J, et al. (2009) The Japanese Society of Hypertension Guidelines for the Management of Hypertension (JSH 2009). Hypertens Res 32: 3-107.

19. Seino Y, Nanjo K, Tajima N, Kadowaki T, Kashiwagi A, et al. (2012) Committee report on the classification and the diagnostic criteria of diabetes mellitus. Diabetes (Tounyoubyo) 55: 485-504 (In Japanese).

20. Omura M, Nishikawa T (2006) Screening tests and diagnostic examinations of hypertension for primary aldosteronism. Rinsho Byori 54: 1157-1163 (In Japanese).

21. Sonoyama $T$, Sone $M$, Miyashita K, Tamura N, Yamahara K, et al. (2011) Significance of adrenocorticotropin stimulation test in the diagnosis of an aldosterone-producing adenoma. J Clin Endocrinol Metab 96: 2771-2778.

22. Akehi Y, Murase K, Nagaishi R, Takenoshita H, Nomiyama T, et al. (2011) The current state and problems of adrenal venous sampling in Fukuoka University Hospital. Clinical Endocrinol (Tokyo) 59: 651-655 (In Japanese).

23. Young WF, Stanson AW, Thompson GB, Grant CS, Farley DR, et al. (2004) Role for adrenal venous sampling in primary aldosteronism. Surgery 136:12271235.

24. Kintscher U, Foryst-Ludwig A, Unger T (2008) Inhibiting angiotensin type 1 receptors as a target for diabetes. Expert Opin Ther Targets 12: 1257-1263.

25. Givertz MM (2001) Manipulation of the renin-angiotensin system. Circulation 104: E14-E18.

26. Rita OD, Hackam DG, Spence JD (2012) Effects of aldosterone on human atherosclerosis: plasma aldosterone and progression of carotid plaque. Can J Cardiol 28: 706-711.

27. Stowasser M (2009) Update in primary aldosteronism. $J$ Clin Endocrinol Metab 94: 3623-3630.

28. Queisser N, Schupp N (2012) Aldosterone, oxidative stress, and NF- $\kappa \mathrm{B}$ activation in hypertension-related cardiovascular and renal diseases. Free Radic Biol Med 53: 314-327.

29. Hillaert MA, Lentjes EG, Kemperman H, van der Graaf Y, Nathoe HM, et al. (2012) Aldosterone, atherosclerosis and vascular events in patients with stable coronary artery disease. Int J Cardio 2012 Jun 22 [Epub ahead of print].

30. Pitt B, Zannad F, Remme WJ, Cody R, Castaigne A, et al. (1999) The effect of spironolactone on morbidity and mortality in patients with severe heart failure. Randomized Aldactone Evaluation Study Investigators.
N Engl J Med 341: 709-717.

31. Nishikawa T, Saito J, Omura M (2007) Prevalence of primary aldosteronism: should we screen for primary aldosteronism before treating hypertensive patients with medication? Endocr J 54: 487-495.

32. Karagiannis A, Tziomalos K, Papageorgiou A, Kakafika AI, Pagourelias ED, et al. (2008) Spironolactone versus eplerenone for the treatment of idiopathic hyperaldosteronism. Expert Opin Pharmacother 9: 509-515.

33. Dietz JD, Du S, Bolten CW, Payne MA, Xia C, et al. (2008) A number of marketed dihydropyridine calcium channel blockers have mineralocorticoid receptor antagonist activity. Hypertension 51: 742-748.

34. Hajjar I, Kotchen TA (2003) Trends in prevalence, awareness, treatment, and control of hypertension in the United States, 1988-2000. JAMA 290: 199-206.

35. Nishizaka MK, Zaman MA, Calhoun DA (2003) Efficacy of low-dose spironolactone in subjects with resistant hypertension. Am J Hypertens 16: 925-930.

36. Fallo F, Veglio F, Bertello C, Sonino N, Della Mea P, et al. (2006) Prevalence and characteristics of the metabolic syndrome in primary aldosteronism. J Clin Endocrinol Metab 91: 454-459.

37. Schwartz GL, Turner ST (2005) Screening for primary aldosteronism in essential hypertension: diagnostic accuracy of the ratio of plasma aldosterone concentration to plasma renin activity. Clin Chem 51: 386-394.

38. Uruno A, Matsuda K, Noguchi N, Yoshikawa T, Kudo M, et al. (2011) Peroxisome proliferator-activated receptor-\{gamma\} suppresses CYP11B2 expression and aldosterone production. J Mol Endocrinol 46: 37-39.

39. Kuris S, Iwasaki T, Mitsuba N, Ishibashi K, Dohi Y, et al. (2012) Impact of electrocardiographic findings for diagnosis of left ventricular hypertrophy in patients with primary aldosteronism. J Renin Angiotensin Aldosterone Syst 14: 51-55.

40. Skov J, Dejgaard A, Frøkiær J, Holst JJ, Jonassen T, et al. (2013) Glucagon-Like Peptide-1 (GLP-1): Effect on kidney hemodynamics and renin-angiotensin-aldosterone system in healthy men. J Clin Endocrinol Metab 98: E664-671.

41. Ralph AD (1980) Hyperkalemia and hyporeninemic hypoaldosteronism. Kidney Int 17: 118-134.

42. Young WF (2007) Primary aldosteronism:renaissance of a syndrome. Clin Endocrinol (Oxf) 66: 607-618.

43. Feraco A, Armani A, Mammi C, Fabbri A, Rosano GM, et al. (2013) Role of mineralocorticoid receptor and renin-angiotensin-aldosterone system in adipocyte dysfunction and obesity. J Steroid Biochem Mol Biol 2013 Feb 28 [Epub ahead of print]. 